\title{
Dielectric Relaxation Phenomena of Polylactic Acid with $\beta$-Crystalline Chitin
}

\author{
Katsuyoshi Shinyama, Toru Oi, and Shigetaka Fujita \\ Department of Electronic and Information Engineering, Hachinohe Institute of Technology, 88-1 Myo Ohbiraki, \\ Hachinohe 031-8501, Japan \\ Correspondence should be addressed to Katsuyoshi Shinyama, shinyama@hi-tech.ac.jp
}

Received 2 December 2011; Accepted 6 February 2012

Academic Editor: Deon Bezuidenhout

Copyright ( $) 2012$ Katsuyoshi Shinyama et al. This is an open access article distributed under the Creative Commons Attribution License, which permits unrestricted use, distribution, and reproduction in any medium, provided the original work is properly cited.

$\beta$-crystalline chitin was added to polylactic acid (PLA), and this PLA was then heat-treated at $100^{\circ} \mathrm{C}$ for one minute. The crystallinity of the heat-treated PLA increased to more than $40 \%$, and its crystallization speed also increased significantly. The temperature dependency of these materials' relative permittivity $\left(\varepsilon_{r}^{\prime}\right)$ and relative dielectric loss factor $\left(\varepsilon_{r}^{\prime \prime}\right)$ was also examined. The dielectric absorption peak value in $\varepsilon_{r}^{\prime \prime}$ curve of the PLA to which chitin was added and was smaller than that of PLA without chitin. Additionally, the Havriliak-Negami relaxation function was used to produce approximation curves for the frequency dependency of $\varepsilon_{r}^{\prime}$ and $\varepsilon_{r}^{\prime \prime}$ of chitin with PLA added at $80^{\circ} \mathrm{C}$. As a result, the relaxation strength $(\Delta \varepsilon)$ of the chitin with PLA added was smaller than that of the PLA without chitin, and the relaxation time $(\tau)$ of the chitin with PLA added was approximately 2.5 times larger than that of the PLA without chitin.

\section{Introduction}

Polylactic acid (PLA) is a type of bioplastic. It is a material made from the corn starch. The authors made a study of the electrical characteristics of PLA to investigate the potential of using PLA as an alternative to petroleum-derived plastics and demonstrated through this study that the electrical resistance and electrical insulation breakdown strength of PLA are equivalent to those of low-density polyethylene, a petroleum-derived plastic, at temperatures ranging from room temperature to around $70^{\circ} \mathrm{C}$ and that, therefore, PLA is a good electrical insulation material $[1,2]$. In addition, the research to be applied to polymer-insulated cable and polymeric membranes with ultralow dielectric performance using the PLA has been reported [3-5]. At temperatures higher than $70^{\circ} \mathrm{C}$, however, the insulation performance of PLA deteriorates due to its poor heat resistance, which is a drawback that remains to be resolved $[1,2]$. The deterioration in insulation performance is attributable to the slow crystallization speed of PLA, and one practicable method for increasing crystallization speed is to combine
PLA with a crystal-nucleating agent into one composite material. By the way, chitin exists in a large quantity, so that it is equal to cellulose on the earth. Although a general chitin is $\alpha$-crystalline chitin, it can be said that $\beta$-crystalline chitin is rich in reactivity, and its degree of activity is higher than $\alpha$-crystalline chitin $[6,7]$. The authors selected biomass resources, made $\beta$-crystalline chitin using the cartilaginous components of a squid, a main local product of Hachinohe, combined this $\beta$-crystalline chitin and PLA into a composite material, and demonstrated the possibility that this composite material-prepared by adding chitin to PLAmay be capable of reducing the effect of temperature and thereby preventing the electrical characteristics of PLA from deteriorating at temperatures higher than $70^{\circ} \mathrm{C}$ [8]. Since the efficacy of chitin in increasing the crystallization speed is less than that of crystal nucleating agent, the question of how the crystallization speed can be increased must be further examined for PLA combined with chitin. In this study, after chitin was added to PLA, the PLA to which the chitin had been added was heat-treated, and thermal analysis was conducted to check the dielectric properties. 


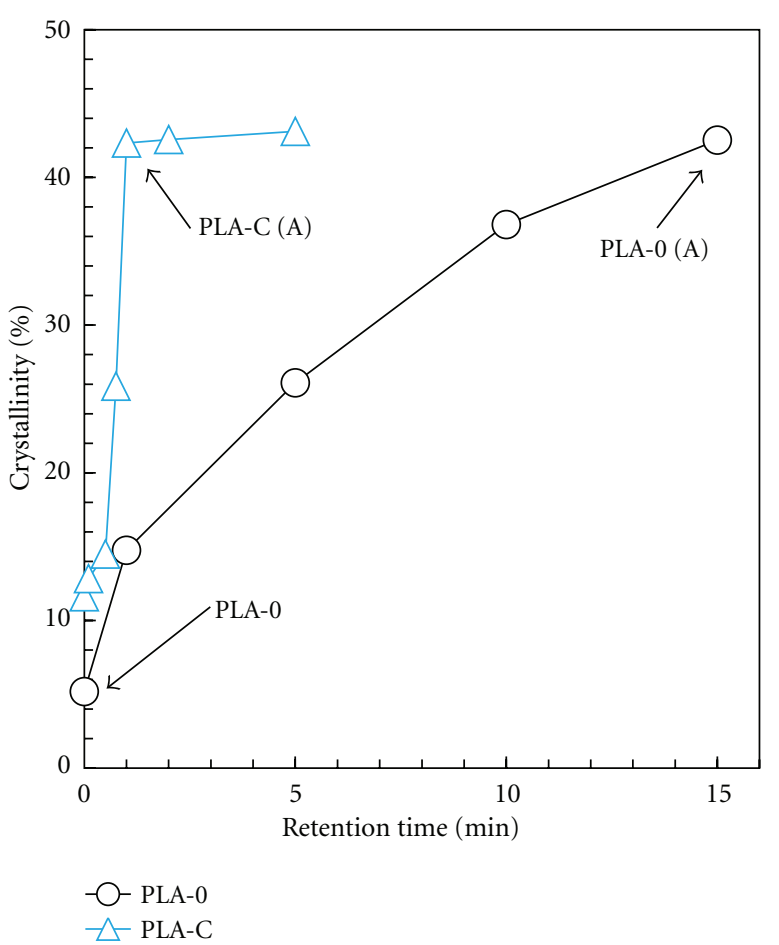

Figure 1: Effect of heat treatment at $100^{\circ} \mathrm{C}$ on crystallinity of PLA with chitin.

\section{Specimen and Experimental Method}

A PLA pellet supplied by Mitsui Chemicals, Inc., was used as the specimen. $1.0 \mathrm{wt} \%$ of chitin, having an average diameter of $3 \mu \mathrm{m}$, was added to this pellet [8]. The weight average molecular weight of PLA is 142,000 . The making method of the specimen used self-nucleation promotion method [9] unlike previous report [8]. The pellet to which chitin had been added was melted, mixed, and kneaded at $190^{\circ} \mathrm{C}$ and then hot-pressed at $200^{\circ} \mathrm{C}$ to form it into a film. Having been formed into a film, the pellet was rapidly cooled to room temperature. In addition, a lot of fine crystalline nucleus formed with specimen controlled the crystallization. Following this, it was heat-treated at $100^{\circ} \mathrm{C}$ for a specified length of time and again rapidly cooled to room temperature. The thickness of this film-shaped specimen was $50 \mu \mathrm{m}$. It was then subjected to thermal analysis using a heat-flux differential scanning calorimeter (DSC). The DSC curve of the PLA was observed while increasing temperature from $20^{\circ} \mathrm{C}$ to $200^{\circ} \mathrm{C}$ at a rate of $10^{\circ} \mathrm{C}$ per minute in a nitrogen atmosphere. After $200^{\circ} \mathrm{C}$ had been reached, the DSC curve of the PLA was observed while decreasing temperature at a rate of $1^{\circ} \mathrm{C}$ per minute. In addition, dielectric properties were measured to verify the electrical characteristics of the PLA after addition of chitin and subsequent exposure to heat treatment. To obtain the dielectric properties, the capacity $(C)$ and the dielectric dissipation factor $(\tan \delta)$ of a specimen were measured simultaneously while heating it at a rate of $1^{\circ} \mathrm{C}$ per minute from $30^{\circ} \mathrm{C}$ to $150^{\circ} \mathrm{C}$, and the relative permittivity $\left(\varepsilon_{r}^{\prime}\right)$ and relative dielectric loss factor $\left(\varepsilon_{r}^{\prime \prime}\right)$, the real part and the imaginary part of complex relative permittivity $\left(\varepsilon_{r}^{*}\right)$, respectively, were obtained. A voltage of 1 Vrms was applied to measure $C$ and $\tan \delta$. A frequency $(f)$ of $1 \mathrm{kHz}$ was used to examine the temperature dependency of $\varepsilon_{r}^{\prime}$ and $\varepsilon_{r}^{\prime \prime}$. Finally, to examine the frequency dependency of $\varepsilon_{r}^{\prime}$ and $\varepsilon_{r}^{\prime \prime}, f$ was varied from $50 \mathrm{~Hz}$ to $1 \mathrm{MHz}$ while keeping the temperature of the specimen at $80^{\circ} \mathrm{C}$.

\section{Experimental Results and Discussion}

3.1. Thermal Analysis. Figure 1 shows the effect of heat treatment at $100^{\circ} \mathrm{C}$ on crystallinity $\left(x_{c}\right)$ of PLA with chitin. The specimen to which chitin has not been added is here called PLA-0, and the one to which chitin has been added is called PLA-C. $x_{c}$ was calculated using (1) below and referring to the DSC curve [10]:

$$
x_{c}(\%)=\frac{\left|\Delta H_{c}+\Delta H_{m}\right|}{\left|\Delta H_{m}(100 \%)\right|} \times 100,
$$

where $x_{c}$ is crystallinity (\%), $\Delta H_{c}$ is crystallization enthalpy $(\mathrm{J} / \mathrm{g}), \Delta H_{m}$ is fusion enthalpy $(\mathrm{J} / \mathrm{g})$, and $\Delta H_{m}(100 \%)$ is fusion enthalpy $(-93 \mathrm{~J} / \mathrm{g})$ when the crystallinity of PLA is $100 \%$. When the heat treatment time was zero, $x_{c}$ of PLA-0 was around $6 \%$. It increased moderately as the heat treatment time increased; specifically, it increased to around $42 \%$ after heat treatment had been conducted for 15 minutes. PLA-0 that has been heat-treated for 15 minutes is here called PLA-0 (A). $x_{c}$ of PLA-C was around $12 \%$ when the heat treatment time was zero. After heat treatment began, it increased rapidly; specifically, it reached about $42 \%$ after only one minute. PLA-C that has been heat-treated for one minute is here called PLA-C(A). As is clear from the results described above, a 15-minute heat treatment is required to increase $x_{c}$ of PLA- 0 to more than $40 \%$, while only a oneminute heat treatment is required to increase $x_{c}$ of PLA-C to more than $40 \%$. This shows that the crystallization speed can be substantially increased by adding chitin and then performing heat treatment. $x_{c}$ remained almost unchanged after it had increased to above $40 \%$ even if further heat treatment was performed. This was observed with all the specimens used in this study.

Figure 2 shows a polarizing microscope image of chitin with PLA added. A magnification of $\times 400$ was used. No spherocrystals were observed in PLA-0, while spherocrystals had grown in PLA-0(A). It is probable that the selfnucleation promotion method first formed in the specimen many tiny spherocrystals nuclei, which the heat treatment caused to grow, so increasing the spherocrystal density. In PLA-C(A), spherocrystals similar to those in PLA-0(A) were also observed. This is probably because the chitin added to PLA caused spherocrystals nuclei to form, from which spherocrystals grew to increase the spherocrystal density. Additionally, cross stripes can be faintly seen in the spherocrystals of PLA-0(A) and PLA-C(A). This is probably because spherocrystals bounced off each other during the spherocrystal growth, halting their growth in the radial direction.

Figure 3 shows the DSC curve when the temperature of the PLA with chitin is decreasing. The DSC curve of PLA$\mathrm{C}$ has been shifted by $-0.3 \mathrm{~W} / \mathrm{g}$ for the sake of visibility in 


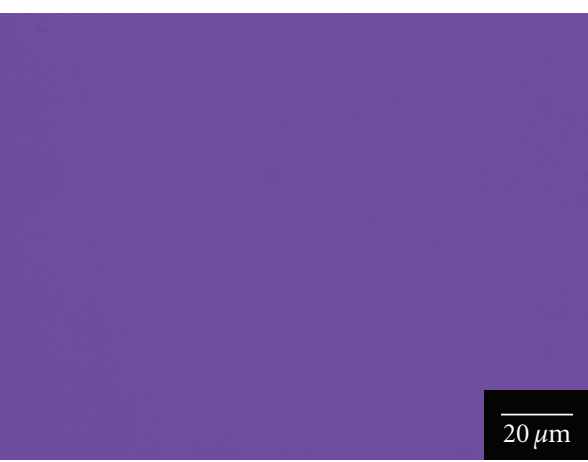

(a) PLA-0

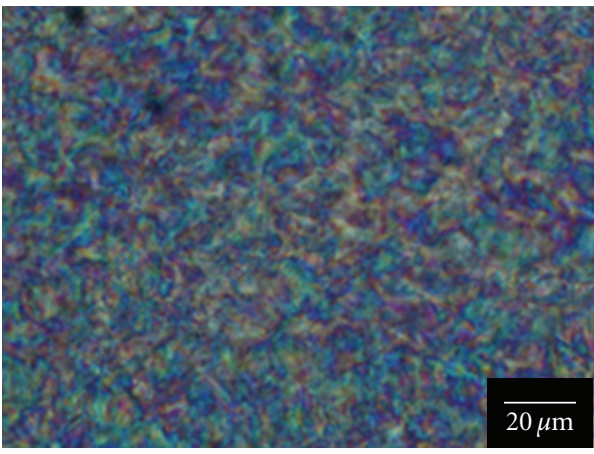

(b) PLA-0A

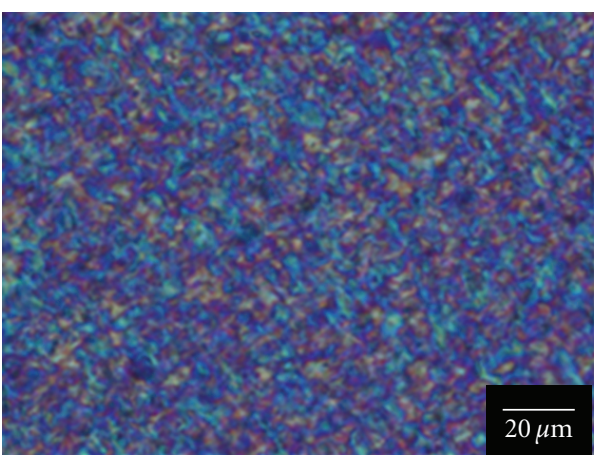

(c) PLA-C(A)

Figure 2: Polarization microscope photograph of PLA with chitin.

this figure. In the case of PLA-0, the crystallization peak was observed at a temperature of around $122^{\circ} \mathrm{C}$. In the case of PLA-C, it was observed at a temperature of around $132^{\circ} \mathrm{C}$; that is, it moved to around $10^{\circ} \mathrm{C}$ higher than for PLA- 0 . From this result, it is considered that the chitin added facilitated the PLA crystallization process, with the chitin itself functioning as a nucleating agent.

3.2. Dielectric Characteristics. Figure 4 shows the temperature dependency of relative permittivity $\left(\varepsilon_{r}^{\prime}\right)$ and relative dielectric loss factor $\left(\varepsilon_{r}^{\prime \prime}\right)$ of chitin with PLA added. The relative permittivity $\left(\varepsilon_{r}^{\prime}\right)$ of PLA-0 sharply increased starting at about $60^{\circ} \mathrm{C}$. Because the glass transition point $\left(T_{g}\right)$ of PLA is about $60^{\circ} \mathrm{C}[1,2]$, dipoles probably aligned at this temperature accompanied by main-chain segments in

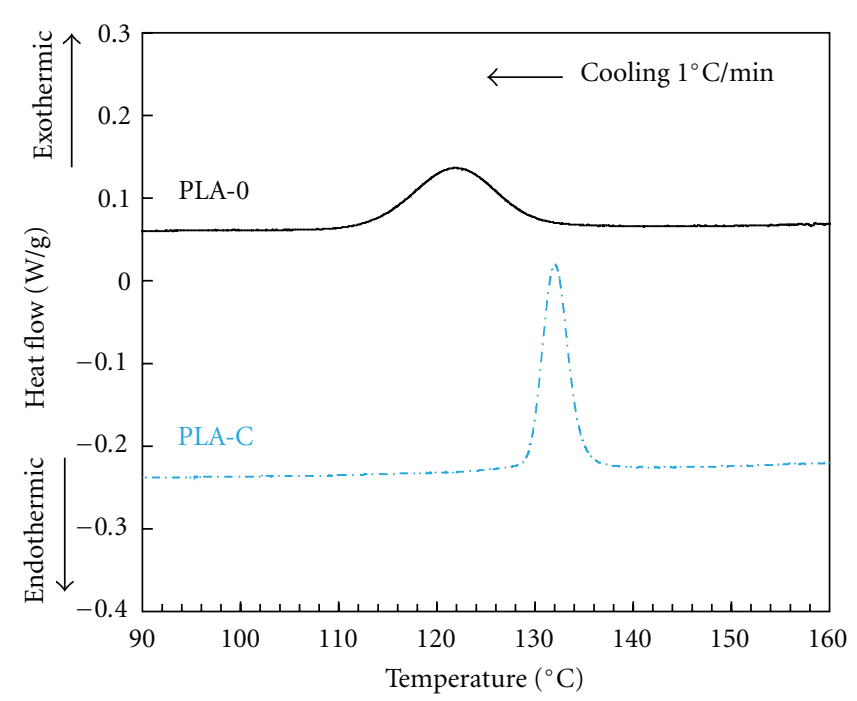

FIGURE 3: DSC curve when temperature of PLA with chitin is decreasing.

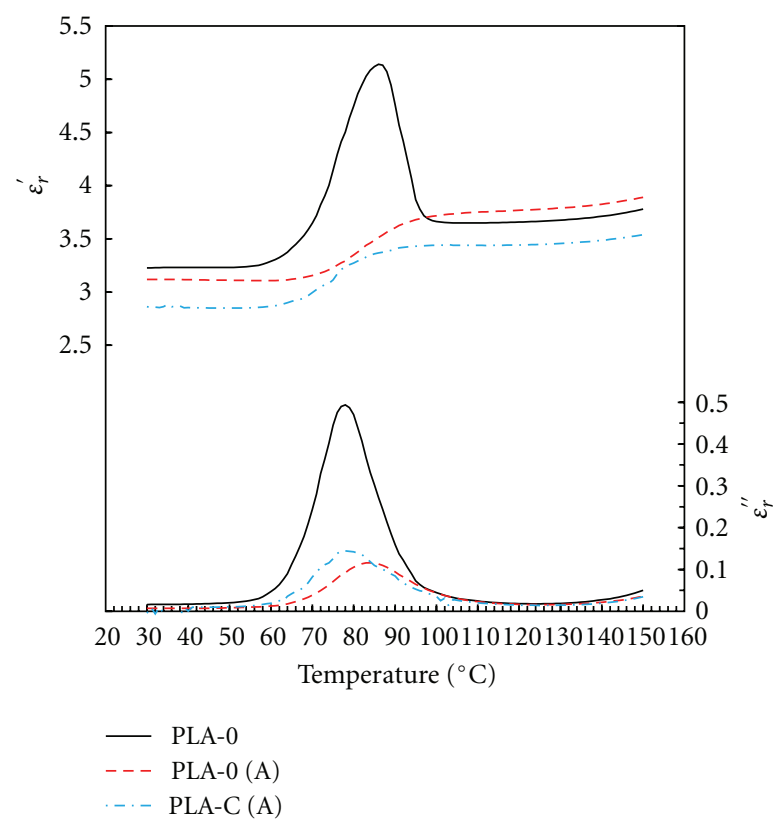

FIGURE 4: Temperature dependence of dependence of $\varepsilon_{r}^{\prime}$ and $\varepsilon_{r}^{\prime \prime}$ of PLA with chitin.

motion that had been thermally activated by the microBrownian motion. PLA- 0 shows a peak in its $\varepsilon_{r}^{\prime}$ curve at about $90^{\circ} \mathrm{C}$, while PLA-O(A) and PLA-C(A) do not show peaks. This peak appears when the specimen crystallizes during the temperature history for measurement [11]. Meanwhile, peak values of $\varepsilon_{r}^{\prime \prime}$ of PLA-O(A) and PLA-C(A) were smaller that of PLA- 0 . This peak value of $\varepsilon_{r}^{\prime \prime}$ of PLA is probably $\alpha$-absorption because it appears higher in the temperature range than $T_{g}$ [12]. Also, because the relaxation time distribution becomes broader for $\alpha$-absorption as the crystallinity of specimen increases, PLA-0(A) and PLA-C(A) are considered to have a broader relaxation time distribution than PLA-0 [13]. 


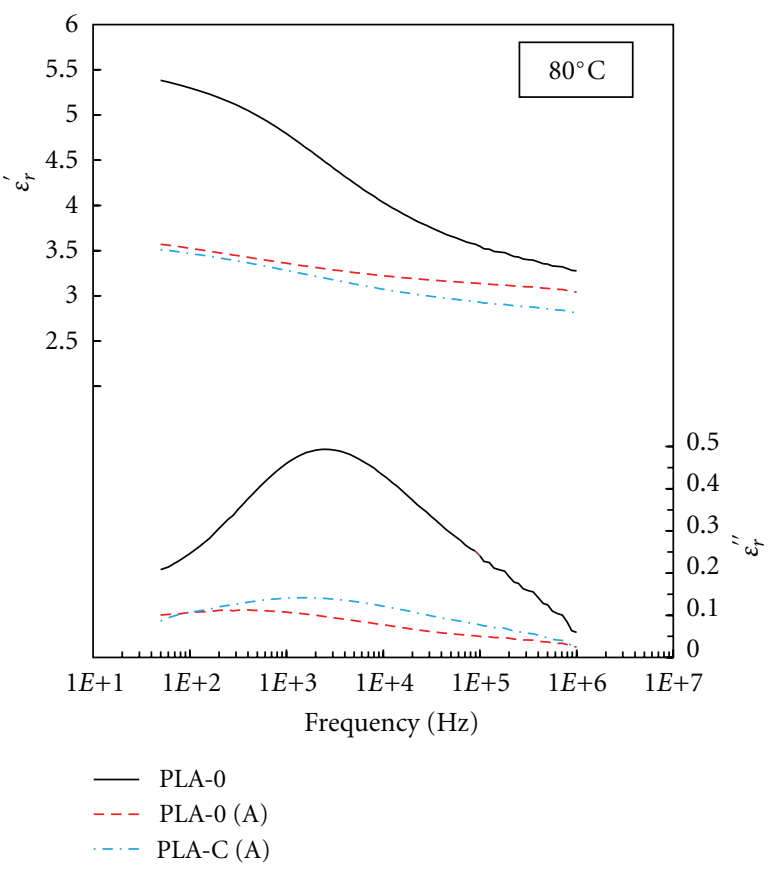

FIGURe 5: Frequency dependence of $\varepsilon_{r}^{\prime}$ and $\varepsilon_{r}^{\prime \prime}$ of PLA with chitin at $80^{\circ} \mathrm{C}$.

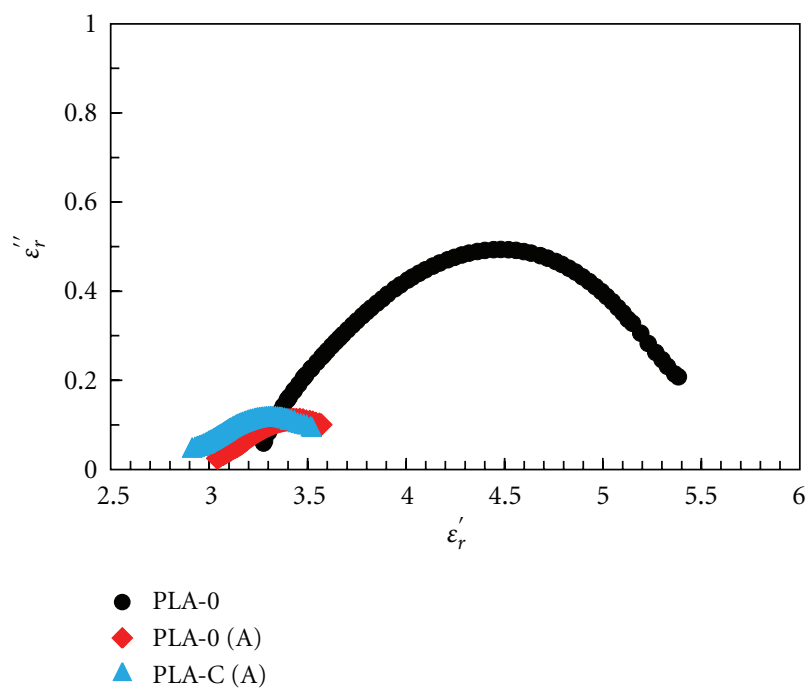

FIgURE 6: Havriliak-Negami type relaxation of PLA with chitin at $80^{\circ} \mathrm{C}$.

Figure 5 shows the frequency dependency of $\varepsilon_{r}^{\prime}$ and $\varepsilon_{r}^{\prime \prime}$ of chitin with PLA added. The measurement was carried out at $80^{\circ} \mathrm{C}$, which is higher than $T_{g}$. For PLA-0, its $\varepsilon_{r}^{\prime}$ decreased as the frequency $(f)$ increased. For PLA-0(A), although, like PLA-0, its $\varepsilon_{r}^{\prime}$ decreased as $f$ increased, it showed a smaller decrease rate than that of PLA-0. The curve of $\varepsilon_{r}^{\prime}$ for PLA$\mathrm{C}(\mathrm{A})$ was similar to that of PLA-0(A). Additionally, the dielectric absorption peak value in the $\varepsilon_{r}^{\prime \prime}$ curve of PLA-0 maximized at about $3 \mathrm{kHz}$, while the dielectric absorption peak values for PLA-0(A) and PLA-C(A) maximized at about
TABle 1: Values of $\varepsilon_{r \infty}, \varepsilon_{r 0}, \Delta \varepsilon, \alpha, \beta$, and $\tau$ at $80^{\circ} \mathrm{C}$.

\begin{tabular}{lcccccc}
\hline & $\varepsilon_{r \infty}$ & $\varepsilon_{r 0}$ & $\Delta \varepsilon$ & $\alpha$ & $\beta$ & $\tau(\mu \mathrm{sec})$ \\
\hline PLA-0 & 2.94 & 5.57 & 2.31 & 0.26 & 0.51 & 63.7 \\
PLA-0(A) & 2.99 & 3.90 & 0.87 & 0.24 & 0.36 & 127.4 \\
PLA-C(A) & 2.75 & 3.74 & 0.86 & 0.16 & 0.35 & 159.0 \\
\hline
\end{tabular}

$500 \mathrm{~Hz}$, which is lower than that for PLA-0. And these peak values were smaller than that for PLA- 0 .

Maeno et al. have reported that the Havriliak-Negami relaxation can approximate the complex relative permittivity of PLA [14]. The complex permittivity $\left(\varepsilon_{r}^{*}\right)$ of a polymer can be expressed with the Havriliak-Negami relaxation function shown in (2) and (3) below, where $\varepsilon_{0}$ and $\varepsilon_{\infty}$ are limiting lowand high-frequency dielectric constants; respectively, $\omega$ is the angular frequency, $\tau_{0}$ is the mean relaxation time, and $\alpha$ and $\beta$ are distribution parameters [15]:

$$
\begin{gathered}
\varepsilon_{r}{ }^{*}=\varepsilon_{r \infty}+\frac{\Delta \varepsilon}{\left(1+(i \omega \tau)^{\beta}\right)^{\alpha}}, \\
\Delta \varepsilon=\varepsilon_{r 0}-\varepsilon_{r \infty} .
\end{gathered}
$$

The authors found that the results shown in Figure 5 were approximated by (2). The dielectric parameters calculated from the data shown in Figure 6 are given in Table 1. Figure 6 shows a plot of the $\varepsilon_{r}^{\prime}$ and $\varepsilon_{r}^{\prime \prime}$ at $85^{\circ} \mathrm{C}$, with a dotted line showing an approximated curve. As shown in this figure, the authors were generally able to fit the actual measurements to the approximation curve.

For PLA-0, $\Delta \varepsilon$ is 2.31, while, for PLA-0(A) and PLA$\mathrm{C}(\mathrm{A})$, it is between 0.86 and 0.87 , smaller than that for PLA-0. As seen from Figure 1, because the heat treatment increased $x_{c}$ values of PLA-0(A) and PLA-C (A), plate crystals (lamellar crystals) were probably formed, and these lamellar crystals connected to each other via tie molecules, and then molecular chains in the amorphous regions were constrained. In other words, the heat treatment probably inhibited the dipole alignment to make $\Delta \varepsilon$ for PLA-0(A) and PLA-C(A) smaller than that for PLA-0 [11, 15]. Next, $\beta$ for PLA-0 is 0.51 , while, for PLA-0(A) and PLA-C(A), it is between 0.35 and 0.36 , smaller than that for PLA-0. In other words, the heat treatment broadened the relaxation time distribution, which is consistent with what was derived from the temperature dependency of $\varepsilon_{r}^{\prime \prime}$ in Figure 4. Therefore, the increase in $x_{c}$ probably broadened the relaxation time distribution.

The relaxation time $(\tau)$ of PLA- 0 is $63.7 \mu$ s, while that of PLA- $0(\mathrm{~A})$ is $127.4 \mu \mathrm{s}$, which is about twice that of PLA0 . And $\tau$ of PLA-C(A) is $159.0 \mu \mathrm{s}$, which is about 2.5 times that of PLA-0. The reason for this is considered to be that, as the reason for the decrease in $\Delta \varepsilon$, the increase in $x_{c}$ of the specimen inhibited dipole alignment, lengthening $\tau$.

\section{Summary}

The crystallization speed of PLA was substantially increased by adding $1 \mathrm{wt} \%$ of $\beta$-crystalline chitin to PLA and then 
heat-treating this PLA at $100^{\circ} \mathrm{C}$ for one minute. Also, the temperature dependency of these materials' relative permittivity $\left(\varepsilon_{r}^{\prime}\right)$ and relative dielectric loss factor $\left(\varepsilon_{r}^{\prime \prime}\right)$ were examined. The dielectric absorption peak value in $\varepsilon_{r}^{\prime \prime}$ curve of the PLA to which chitin was added (PLA-C $(A))$, was close to that of heat-treated PLA (PLA-0(A)), and smaller than that of PLA without chitin (PLA-0). Additionally, the Havriliak-Negami relaxation function was used to produce approximation curves for the frequency dependency of $\varepsilon_{r}^{\prime}$ and $\varepsilon_{r}^{\prime \prime}$ of chitin with PLA added at $80^{\circ} \mathrm{C}$. As a result, the relaxation strength $(\Delta \varepsilon)$ of PLA-C $(\mathrm{A})$ resembled that of PLA0 (A) and was smaller than that of PLA-0. Moreover, the relaxation time $(\tau)$ of PLA-C $(\mathrm{A})$ was approximately 2.5 times larger than that of PLA-0.

\section{Acknowledgments}

The authors would like to express their gratitude to the people at Mitsui Chemicals, Inc. who provided them with PLA samples. Finally, this study was partly supported by grant funding from the 2011 special research (research project) grant program of the Hachinohe Institute of Technology.

\section{References}

[1] K. Shinyama and S. Fujita, "Electrical conduction and dielectric properties of biodegradable plastics," IEEJ Transactions on Fundamentals and Materials, vol. 125, no. 3, pp. 204-208, 2005 (Japanese).

[2] K. Shinyama and S. Fujita, "Mechanical and dielectric breakdown properties of biodegradable plastics," IEEJ Transactions on Fundamentals and Materials, vol. 126, no. 1, pp. 31-36, 2006.

[3] T. Nakagawa, T. Nakiri, R. Hosoya, and Y. Tajitsu, "Electrical properties of biodegradable polylactic acid film," IEEE Transactions on Industry Applications, vol. 40, no. 4, pp. 1020-1024, 2004.

[4] T. Nakiri, Y. Kawachi, M. Honda, K. Imoto, T. Yamatika, and Y. Tajitsu, "Development of electric wire using biodegradable polymer," IEEE Transactions on Industry Applications, vol. 43, no. 4, pp. 1069-1074, 2007.

[5] S. J. Liu, L. Y. Chiou, and J. Y. Liao, "Ultralow dielectric property of electrospun polylactide-polyglycolide nanofibrous membranes," Japanese Journal of Applied Physics, vol. 50, no. 10, pp. 10PG05-10PG05-5, 2011.

[6] L. Ren and S. Tokura, "Structural aspects of poly(methyl methacrylate)-grafted $\beta$-chitin copolymers initiated by ceric salt," Carbohydrate Polymers, vol. 23, no. 1, pp. 19-25, 1994.

[7] H. Sashiwa, S. Fujishima, N. Yamano et al., "Production of Nacetyl-D-glucosamine from $\beta$-chitin by enzymatic hydrolysis," Chemistry Letters, vol. 30, no. 4, pp. 308-309, 2001.

[8] K. Shinyama and S. Fujita, "Development of environmentally conscious insulating material using biomass resources," in Proceedings of the 38th Symposium Electrical and Electronic Insulating Materials and Applications in Systems, pp. 155-158, 2007.

[9] H. Tsuji, Poly-Lactic Acid, Yoneda Shuppan, Tokyo, Japan, 2008.

[10] H. Tsuji and Y. Ikada, Poly-Lactic Acid, Kobunshi Kankokai, Tokyo, Japan, 1997.
[11] T. Oi, K. Shinyama, and S. Fujita, "Electrical properties of heat-treated poly-lactic acid," IEEJ Transactions on Fundamentals and Materials, vol. 131, no. 5, pp. 395-400, 2011 (Japanese).

[12] K. Shinyama and S. Fujita, "Mechanical and electrical characteristics of poly-lactic acid which added plasticizer," IEEJ Transactions on Fundamentals and Materials, vol. 130, no. 4, pp. 369-376, 2010 (Japanese).

[13] Y. Inuishi, T. Nakajima, K. Kawabe, and M. Ieda, Phenomenological Dielectrics Theory, The Institute of Electrical Engineers of Japan, 1973.

[14] Y. Maeno, Y. Yamaguchi, N. Hirai et al., "Effect of glass transition on electriccal conduction characteristics of poly-Llactic acid," IEEJ Transactions on Fundamentals and Materials, vol. 125, no. 3, pp. 254-260, 2005 (Japanese).

[15] E. Fukuda et al., Physics of Polymers, Asakura, 1963. 

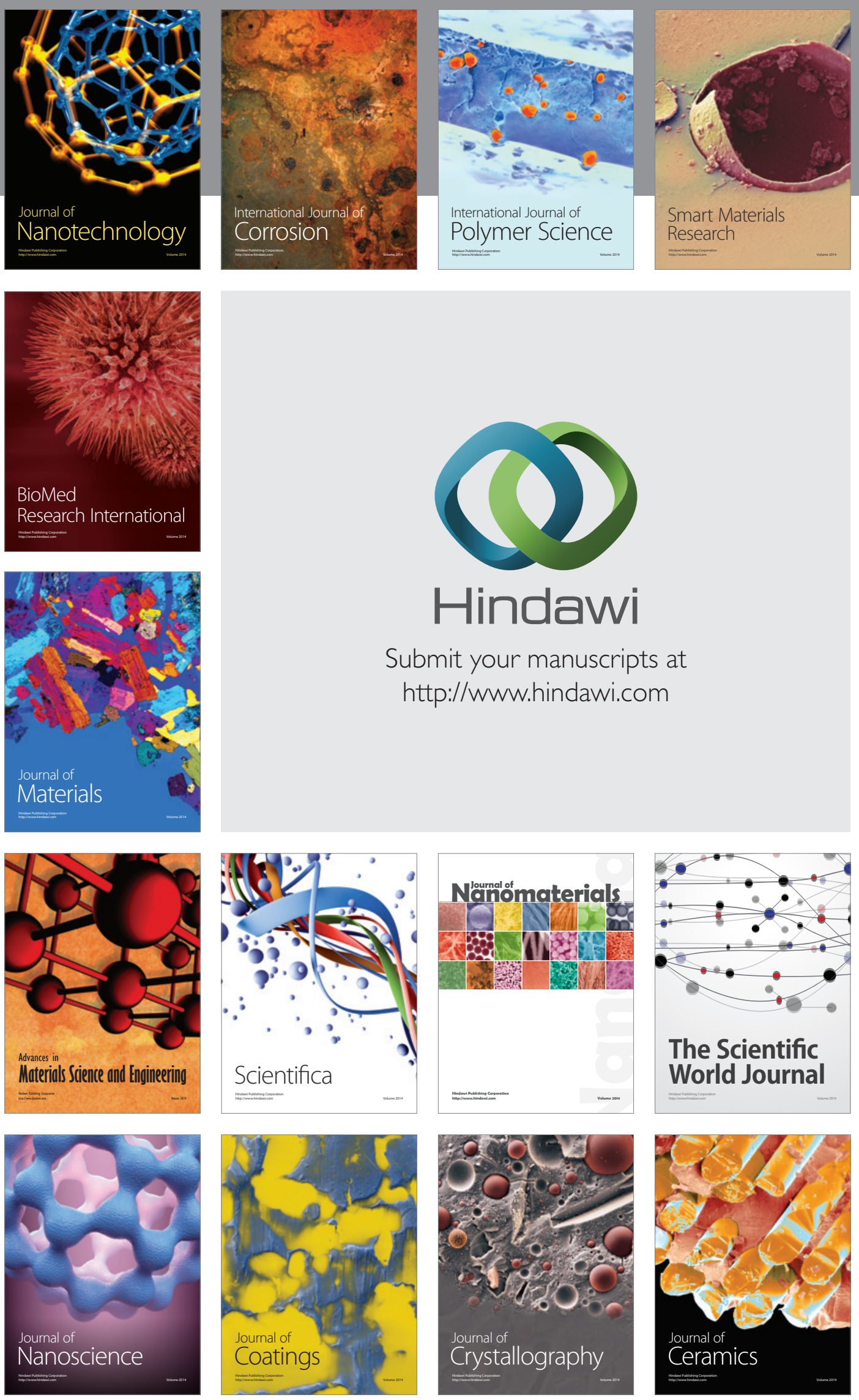

The Scientific World Journal

Submit your manuscripts at

http://www.hindawi.com

\section{World Journal}

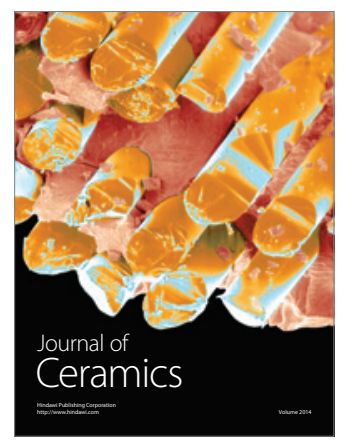

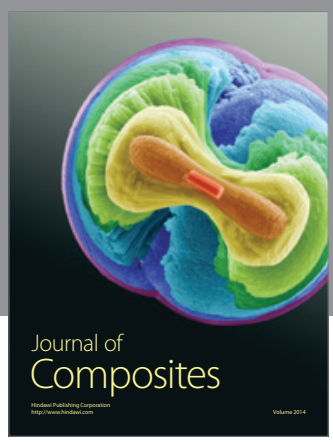
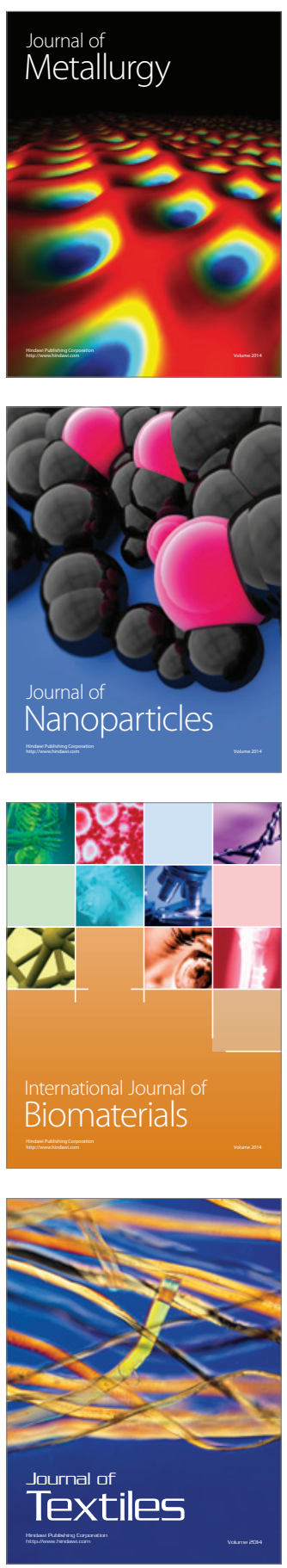